\title{
Safety and Efficacy of C-reactive Protein-guided Antibiotic Use to Treat Acute Respiratory Infections in Tanzanian Children: A Planned Subgroup Analysis of a Randomized Controlled Noninferiority Trial Evaluating a Novel Electronic Clinical Decision Algorithm (ePOCT)
}

\author{
Kristina Keitel, ${ }^{1,2, \odot}$ Josephine Samaka, ${ }^{3,4}$ John Masimba, ${ }^{3}$ Hosiana Temba, ${ }^{3}$ Zamzam Said, ${ }^{3}$ Frank Kagoro, ${ }^{3}$ Tarsis Mlaganile, ${ }^{3}$ Willy Sangu, ${ }^{5}$ Blaise \\ Genton, ${ }^{1,6}$ and Valerie D'Acremont ${ }^{1,7}$ \\ ${ }^{1}$ Swiss Tropical and Public Health Institute, Basel, and ${ }^{2}$ Department of Pediatric Emergency Medicine, University Hospital Bern, Switzerland; ${ }^{3}$ Ifakara Health Institute, ${ }^{4}$ Amana Hospital, and ${ }^{5}$ City \\ Council, Dar es Salaam, Tanzania; and ${ }^{6}$ Infectious Diseases Service, and ${ }^{7}$ Department of Ambulatory Care and Community Medicine, University Hospital Lausanne, Switzerland
}

\section{(See the Editorial Commentary by McIntosh on pages 1935-36.)}

Background. The safety and efficacy of using C-reactive protein (CRP) to decide on antibiotic prescription among febrile children at risk of pneumonia has not been tested.

Methods. This was a randomized (1:1) controlled noninferiority trial in 9 primary care centers in Tanzania (substudy of the ePOCT trial evaluating a novel electronic decision algorithm). Children aged 2-59 months with fever and cough and without life-threatening conditions received an antibiotic based on a CRP-informed strategy (combination of CRP $\geq 80 \mathrm{mg} / \mathrm{L}$ plus age/temperature-corrected tachypnea and/or chest indrawing) or current World Health Organization standard (respiratory rate $\geq 50$ breaths/ minute). The primary outcome was clinical failure by day (D) 7; the secondary outcomes were antibiotic prescription at D0, secondary hospitalization, or death by D30.

Results. A total of 1726 children were included (intervention: 868, control: 858; 0.7\% lost to follow-up). The proportion of clinical failure by D7 was $2.9 \%(25 / 865)$ in the intervention arm vs $4.8 \%$ (41/854) in the control arm (risk difference, $-1.9 \%$ [95\% confidence interval $\{\mathrm{CI}\},-3.7 \%$ to $-.1 \%$ ]; risk ratio [RR], 0.60 [95\% CI, .37-.98]). Twenty of $865(2.3 \%)$ children in the intervention arm vs 345 of $854(40.4 \%)$ in the control arm received antibiotics at D0 (RR, 0.06 [95\% CI, .04-.09]). There were fewer secondary hospitalizations and deaths in the CRP arm: $0.5 \%$ (4/865) vs $1.5 \%(13 / 854)$ (RR, 0.30 [95\% CI, .10-.93]).

Conclusions. CRP testing using a cutoff of $\geq 80 \mathrm{mg} / \mathrm{L}$, integrated into an electronic decision algorithm, was able to improve clinical outcome in children with respiratory infections while substantially reducing antibiotic prescription.

Clinical Trials Registration. NCT02225769.

Keywords. C-reactive protein; respiratory infection; pediatrics; electronic decision support algorithm; integrated management of childhood illness.

Respiratory tract infections are the most important drivers of antibiotic overuse at the primary care level [1]. Overprescription is particularly common in low-resource settings where health workers lack adequate diagnostic support [1]. At the same time, childhood mortality from respiratory infections remains high [2]. With increasing pneumococcal and Haemophilus influenzae type $\mathrm{b}$ vaccine coverage, only a small proportion of

Received 24 September 2018; editorial decision 16 December 2018; accepted 30 January 2019; published online February 2, 2019.

Correspondence: K. Keitel, Swiss Tropical and Public Health Institute, Socinstrasse 57, 4051 Basel, Switzerland (kristina.keitel@swisstph.ch).

Clinical Infectious Diseases ${ }^{\circledR} \quad$ 2019;69(11):1926-34

(C) The Author(s) 2019. Published by Oxford University Press for the Infectious Diseases Society of America. All rights reserved. For permissions, e-mail: journals.permissions@oup.com. DOI: $10.1093 /$ cid/ciz080 these children require antibiotic treatment $[3,4]$. Overrelying on antibiotics has resulted in the neglect of appropriate supportive management of viral respiratory infections [5]. The World Health Organization (WHO) Integrated Management of Childhood Illnesses (IMCI) guidelines currently recommend antibiotic treatment for respiratory infections among children aged 2 months to 5 years based on clinical features only [6]. With the exception of wheezing, clinical features, including respiratory rate, were largely uniform across the spectrum of microbiological etiology $[7,8]$. C-reactive protein (CRP) testing, available as a point-of-care test (POCT), has been proposed as a diagnostic for bacterial pneumonia and to improve primary care antibiotic stewardship $[9,10]$. However, studies on the usefulness of CRP as a diagnostic tool in children, and in low-resource settings, are lacking. 
We developed a 2-step approach for the diagnosis of bacterial pneumonia at outpatient level as part of a novel, tablet-based, electronic decision algorithm (ePOCT), aimed at supporting health workers in the management of febrile children at the primary care level [11]. In a randomized noninferiority trial conducted among febrile children in Tanzania, when compared with an electronic IMCI-based algorithm (New Algorithm for Managing Childhood Illness Using Mobile Technology [ALMANACH]), ePOCT was found to reduce antibiotic prescription by two-thirds and lead to improved clinical outcomes [11]. The components of ePOCT for diagnosing respiratory infections differed substantially from the current WHO-recommended diagnostic approach. The ePOCT protocol included a planned subgroup analysis of children with febrile respiratory infections specifically, to compare antibiotic prescribing and clinical outcomes using a novel CRP-informed diagnostic approach within the ePOCT algorithm, compared with the current WHO diagnostic and treatment approach. Within ePOCT we defined lower respiratory tract infection (LRTI) broadly (cough, fever, and age- and temperature-corrected respiratory rate cutoffs) and, within this category, bacterial LRTI by a high point-of-care (POC) CRP level ( $\geq 80 \mathrm{mg} / \mathrm{L})$.

\section{METHODS}

\section{Study Design}

This was a planned subgroup analysis of children with nonsevere respiratory symptoms within the larger randomized open, controlled noninferiority trial that compared ePOCT to a validated algorithm derived from IMCI (ALMANACH) for managing febrile children aged $<5$ years at 9 public outpatient clinics in Dar es Salaam, Tanzania [11], the methods and results of which have been published previously [11]. The primary study also included a routine care arm that was not used for this subgroup analysis as inclusion criteria for this analysis were not systematically recorded by routine clinicians. This study was approved by the Institutional Review Board of the Ifakara Health Institute and by the National Institute for Medical Research Review Board in Tanzania, the Ethikkommission beider Basel in Switzerland, and by the Boston Children's Hospital Ethical Review Board. Written consent forms were used. A data and safety monitoring committee provided oversight. The trial was performed in accordance with the principles of the Declaration of Helsinki.

\section{Participants}

Consecutive patients aged 2-59 months with fever (axillary temperature $\geq 37.5^{\circ}$ ) presenting during normal business hours were screened for eligibility. Detailed inclusion and exclusion criteria for the main trial are listed in the Supplementary Appendix. For this subgroup analysis, we included all children with febrile nonsevere respiratory symptoms, defined as cough with fever but without signs of severe illness (convulsion or positive meningeal signs, hypoxemia, cyanosis, severe respiratory distress, unable to tolerate oral liquids, severe dehydration, severe anemia, and severe acute malnutrition).

\section{Randomization and Intervention}

Patients were individually randomized to one of the 2 management arms in blocks of 4 according to a computer-generated list. Sealed, opaque forms were used for allocation concealment and opened only after patient enrollment. Patients received clinical management with a study clinician using either ePOCT [11] (intervention arm) or ALMANACH [3] (control arm). Study clinicians were asked to fully adhere to the respective algorithm. The ePOCT and ALMANACH algorithms for managing children with nonsevere respiratory infections are summarized in Figure 1. Admitted patients were managed at the discretion of the hospital personnel. Laboratory POC tests were performed on site according to the recommendation of the relevant algorithm. A malaria rapid diagnostic test (mRDT) was done for all patients in both arms using either SD BIOLINE Malaria Ag P.f/ Pan (Standard Diagnostics) or CareStart Malaria HRP2 (Access Bio). CRP testing was conducted using a semiquantitative lateral-flow test (bioNexia CRPplus, bioMérieux). Voluntary screening for human immunodeficiency virus (HIV) using antibody testing (Determine HIV-1/2, Alere) was offered to all patients in both arms.

Caregivers were asked to return with the child for a scheduled visit on day (D) 3 postrandomization, and at any time if concerned about the child's condition. Patients cured at D3 were followed up by phone only on D7. Patients not cured at D3 were managed at follow-up using the assigned algorithm, and were requested to return at D7. Patients not cured at D7 were treated per the clinician's judgment and an additional follow-up visit was performed at D14. All patients were followed-up by phone at D30 to ascertain interim hospitalizations and vital status.

\section{Outcomes}

The primary outcome measure was the proportion of children with clinical failure by D7 (see definition in Table 1). Secondary outcome measures were the proportion of children prescribed an antibiotic at D0 and between D1 and D6, and of severe adverse events (secondary hospitalizations and death) by D30. At follow-up, clinicians who were unaware of the criteria for defining study outcomes systematically assessed and recorded patient clinical and treatment characteristics, which were used to determine outcomes based on prespecified criteria. The study outcomes were not used to inform patient management.

\section{Statistical Analysis}

To determine noninferiority, the lower limit of the confidence interval (CI) of the primary outcome measure was to be within 3\%, 

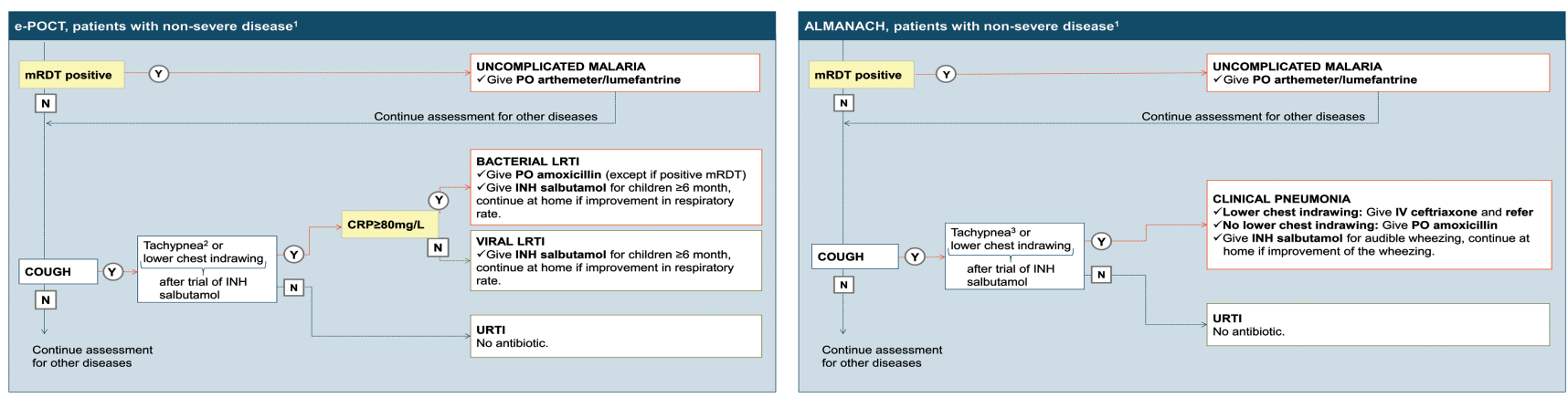

Figure 1. Logic of electronic decision algorithm (ePOCT) and New Algorithm for Managing Childhood Illness Using Mobile Technology (ALMANACH) decision algorithms in managing children with nonsevere respiratory complaints. Questions and information requested by the algorithm are shown on the left side of each panel, and the respective disease classifications and treatment recommendations on the right. Patients in the ePOCT arm received an antibiotic based on a C-reactive protein (CRP)-informed strategy (combination of CRP $\geq 80 \mathrm{mg} / \mathrm{L}$ plus age/temperature-corrected tachypnea and/or chest indrawing); control patients per current World Health Organization (WHO) standard (respiratory rate $\geq 50$ breaths/minute and/or chest indrawing). As an antibiotic treatment, all children from the intervention and control arms (besides 8 children with chest indrawing in the ALMANACH arm who, based on the decision algorithm, were given intramuscular ceftriaxone) were prescribed amoxicillin at 80-100 mg/kg/day for 5 days unless another concomitant infection (eg, skin infection) required broadening of antibiotic coverage. All febrile patients are tested by malaria rapid diagnostic test (mRDT): the main symptoms are all assessed in parallel (eg, a patient with cough is also assessed for the presence of dehydration, skin infection). Within ePOCT, nonsevere children with positive mRDT were not given antibiotics as malaria infection is associated with elevated CRP values, and a study on etiologies of fever performed in the same patient population showed that only 1 among 1005 children had concomitant malaria infection and radiological pneumonia [12]. Inhaled salbutamol treatment was given to all children $>6$ months of age with lower respiratory tract infection. If an improvement in respiratory symptoms was observed (decrease in respiratory rate or resolution of chest indrawing), ePOCT recommended salbutamol prescription for home treatment. Improvement after salbutamol treatment was not used as a criterion for antibiotic prescription. In the ALMANACH arm, inhaled salbutamol treatment was given for patients with auditory wheezing and if an improvement was observed, no antibiotic was prescribed, as recommended in the WHO Integrated Management of Childhood IIIness guidelines. In the latter case, salbutamol was prescribed as a home treatment. Abbreviations: INH, inhaled; IV, intravenous; LRTI, lower respiratory tract infection; mRDT, malaria rapid diagnostic test; N, no; PO, per os; URTI, upper respiratory tract infection; Y, yes.

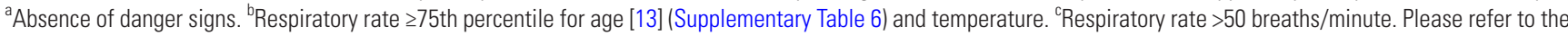
main publications of ePOCT and ALMANACH for details on the content of the entire algorithm [11].

which was established based on what was considered a clinically meaningful difference in clinical failure by D7 [11]. In the subgroup analysis, for the observed clinical failure rate of $4.8 \%$ in the control arm and total sample size of 1726 subjects, we had $81 \%$ power to determine a difference of this magnitude. With the

\section{Table 1. Definition of Clinical Failure by Day 7 (Primary Outcome Measure)}

\section{At Any Time}

Between Initial

Assessment and

Day 7

At Day 3

\begin{tabular}{|c|c|c|}
\hline $\begin{array}{l}\text { - Severe disease: } \\
\text { - Coma } \\
\text { - >2 convulsions } \\
\text { within } 24 \mathrm{~h} \\
\text { - Inability to drink or } \\
\text { breastfeed } \\
\text { - Hypoxemia } \\
\left(\mathrm{SaO}_{2}<90 \%\right) \\
\text { - Severe tachypnea } \\
\text { - Severe tachy- } \\
\text { cardia }^{\text {b }}\end{array}$ & $\begin{array}{l}\text { - Clinical } \\
\text { pneumonia: } \\
\text { - Cough and } \\
\text { tachypnea }^{c} \\
\text { - Cough and } \\
\text { lower chest } \\
\text { indrawing } \\
\text { - Significant } \\
\text { dehydration }\end{array}$ & $\begin{array}{l}\text { - Fever or temperature } \geq 38^{\circ} \mathrm{C} \\
\text { - Clinical pneumonia: } \\
\text { - Cough and tachypnea } \\
\text { - Cough and lower chest } \\
\text { indrawing } \\
\text { - Diarrhea } \\
\text { - Significant dehydration }{ }^{\mathrm{d}} \\
\text { - Serious skin infection } \\
\text { - A new significant symptom or } \\
\text { sign related to the acute episode } \\
\text { but not present at day } 0 \text {. }\end{array}$ \\
\hline
\end{tabular}

Abbreviation: $\mathrm{SaO}_{2}$, oxygen saturation.

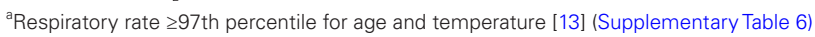
${ }^{b}$ Heart rate $\geq 90$ th percentile for age and temperature [14].

${ }^{c}$ Respiratory rate $\geq 60$ breaths/minute and age $<12$ months or respiratory rate $\geq 50$ breaths/ minute and age $\geq 12$ months.

${ }^{\mathrm{d} D e h y d r a t i o n}$ requiring facility-based treatment.

${ }^{\mathrm{e}}$ Three or more liquid stools per day.

${ }^{\text {fS}}$ kin infection requiring systemic antibiotic treatment and/or facility-based treatment. observed proportions of clinical failure, and an a level of .05, we had $100 \%$ power to detect a difference in clinical failures between the 2 groups. The modified intention-to-treat (mITT) population comprised all randomized patients for which the inclusion criteria for the subgroup analysis was assessed (all patients for whom data were entered into the algorithm on D0). Patients who were lost to follow-up were considered clinical failures. The per-protocol (PP) population included all randomized patients who received the intervention or control procedures and completed the D7 assessment (Figure 2). The primary outcome was calculated for both the mITT and PP populations. Risk difference (RD) and risk ratio (RR) values with $95 \%$ CIs were calculated to estimate the crude intervention effects on the main study outcomes using the Stata cs procedure. Stratified analyses with Mantel-Haenszel estimates for RR were performed to explore heterogeneity of the effect by clinicians. For the primary outcome, mixed-effects logistic regression was used to adjust for possible confounding factors and to account for the hierarchical nature of the data. Odds ratios (ORs) were interpreted as RR since the primary outcome was rare. We used Stata version 15.1.

\section{RESULTS}

\section{Study Population}

From 10 December 2014 to 10 February 2016, a total of 1726 febrile patients with nonsevere respiratory symptoms were included: 868 (50\%) were allocated to ePOCT, and 858 (50\%) to 


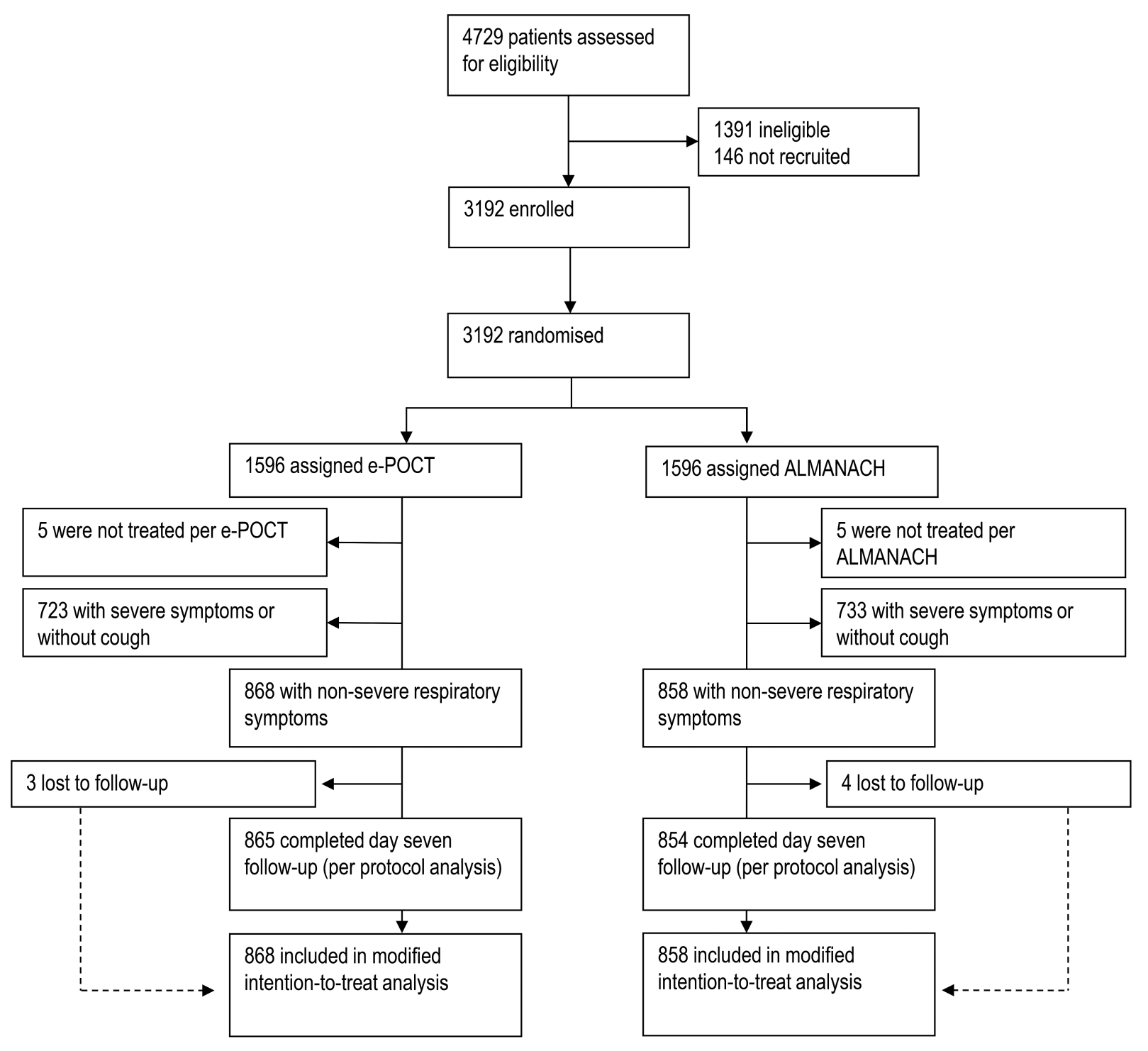

Figure 2. Trial profile. Abbreviations: ALMANACH, New Algorithm for Managing Childhood Illness Using Mobile Technology.

ALMANACH. The groups were balanced with respect to baseline characteristics (Table 2). Three and 4 patients were lost to follow-up before the D7 outcome assessment in the intervention and control arms, respectively. The D30 phone follow-up was completed in $99 \%(852 / 865)$ of patients in the ePOCT arm and $99 \%(844 / 854)$ of patients in the ALMANACH arm.

\section{Primary and Secondary Outcomes}

Twenty-five of 865 (2.9\%) patients experienced clinical failure by D7 in the ePOCT arm vs 41 of 854 (4.8\%) in the ALMANACH arm (RD, $-1.9 \%$ [95\% CI, $-3.7 \%$ to -.1\%]; RR, 0.60 [95\% CI, .37-.98]; Table 3). The main difference in clinical outcome between the 2 arms occurred before D3 (RR for clinical failure, 0.33 [95\% CI, .17-.72]; Supplementary Table 2$) ; 0.2 \%(2 / 865)$ of patients in the ePOCT arm vs $1.4 \%$
$(12 / 854)$ of patients in the ALMANACH arm experienced "severe disease" as per the clinical failure criteria (Table 1) at follow-up (RR, 0.16 [95\% CI, .04-.73]; Supplementary Table 2). At D0, 2.3\% (20/865) of participants in the intervention vs $40.4 \%(345 / 854)$ in the control arm were prescribed an antibiotic treatment ( $\mathrm{RD},-38.0$ [95\% CI, -41.5 to -34.6$]$; RR, 0.06 [95\% CI, .04-.09]; Table 3). Using IMCI (rather than ALMANACH) respiratory rate cutoffs to inform antibiotic treatment, $44 \%$ of patients in the ALMANACH arm would have been prescribed an antibiotic at D0 (Table 2). The risk of secondary admissions or deaths was smaller in the ePOCT arm than the ALMANACH arm: $0.5 \%(4 / 865)$ vs $1.5 \%(13 / 854)$, respectively (RR, 0.30 [95\% CI, .10-.93]; Table 3$)$. The results of the adjusted outcome analysis are displayed in Supplementary Tables 3 and 4. 
Table 2. Baseline Characteristics

\begin{tabular}{|c|c|c|}
\hline Characteristic & ePOCT & ALMANACH \\
\hline \multicolumn{3}{|l|}{ Demographic } \\
\hline Male sex & $486 / 868(56)$ & $465 / 858(54)$ \\
\hline \multicolumn{3}{|l|}{ Age group } \\
\hline $2-11 \mathrm{mo}$ & $387 / 868$ (45) & $405 / 858(47)$ \\
\hline $12-23 \mathrm{mo}$ & 300/868 (35) & $273 / 858(32)$ \\
\hline$\geq 24 \mathrm{mo}$ & $181 / 868(21)$ & $180 / 858(21)$ \\
\hline Received PCV & $443 / 461(96)$ & $451 / 470(96)$ \\
\hline Received Hib vaccine & $447 / 461(97)$ & $456 / 470(97)$ \\
\hline $\begin{array}{l}\text { Primary caregiver other than } \\
\text { mother }\end{array}$ & $40 / 850(5)$ & $41 / 839(5)$ \\
\hline \multicolumn{3}{|l|}{ Mother's highest grade of education } \\
\hline None & $79 / 847$ (9) & $75 / 839(9)$ \\
\hline Primary & $559 / 847(66)$ & $550 / 839(65)$ \\
\hline Postprimary & 209/847 (25) & $215 / 839(26)$ \\
\hline $\begin{array}{l}\text { No. of children in household, me- } \\
\text { dian (IQR) }\end{array}$ & $2(1-3)$ & $2(1-3)$ \\
\hline \multicolumn{3}{|l|}{ Medical history } \\
\hline \multicolumn{3}{|c|}{ Symptoms other than cough and fever } \\
\hline Diarrhea & $95 / 868(11)$ & $138 / 858(16)$ \\
\hline Skin problem & $31 / 868(4)$ & $35 / 858(4)$ \\
\hline \multicolumn{3}{|l|}{ Duration of fever } \\
\hline$\leq 1 \mathrm{~d}$ & $530 / 868(61)$ & $502 / 858(58)$ \\
\hline $2-4 d$ & $327 / 868$ (38) & $344 / 858(40)$ \\
\hline$\geq 5 \mathrm{~d}$ & $11 / 868(1)$ & 12/858 (1) \\
\hline \multicolumn{3}{|l|}{ Duration of cough } \\
\hline$\leq 2 \mathrm{~d}$ & $585 / 847(69)$ & $569 / 844(67)$ \\
\hline $3-6 d$ & 252/847 (30) & 270/844 (32) \\
\hline$\geq 7 \mathrm{~d}$ & 10/847 (1) & $5 / 844(1)$ \\
\hline \multicolumn{3}{|l|}{ Clinical characteristics } \\
\hline WAZ ${ }^{\mathrm{a}}$, mean (SD) & $-0.6(1.2)$ & $-0.6(1.2)$ \\
\hline Respiratory rate, median (IQR) & $41(36-50)$ & $43(36-52)$ \\
\hline Heart rate, mean (SD) & $145(17)$ & $143(16)$ \\
\hline Tachypnea per $\mid \mathrm{MCl}^{\mathrm{b}}$ & $386 / 868(45)$ & $373 / 858(44)$ \\
\hline Lower chest wall indrawing & $7 / 868(1)$ & $8 / 858(1)$ \\
\hline \multicolumn{3}{|l|}{ Laboratory values } \\
\hline mRDT positive & $85 / 868(10)$ & $85 / 858(10)$ \\
\hline HIV-1/2 antibody positive & 10/807 (1) & $6 / 794(1)$ \\
\hline
\end{tabular}

Data are presented as no./No. (\%) unless otherwise indicated.

Abbreviations: ALMANACH, New Algorithm for Managing Childhood Illness Using Mobile Technology; Hib; Haemophilus influenzae b; HIV, human immunodeficiency virus: $I \mathrm{MCl}$, Integrated Management of Childhood IIIness; IQR, interquartile range; mRDT, malaria rapid diagnostic test; $\mathrm{PCV}$, pneumococcal conjugate vaccine; SD, standard deviation; WAZ weight-for-age $z$ score.

aWorld Health Organization 2006 growth curve [15]

bess than 12 months of age and respiratory rate $\geq 50$ breaths/minute, or $\geq 12$ months of age and respiratory rate $\geq 40$ breaths/minute. On day 0 , one patient in the ePOCT arm was admitted for a newly diagnosed HIV infection; in the ALMANACH arm, 7 were admitted for the presence of lower chest wall indrawing without concomitant signs of severe disease (as per $2008 \mathrm{IMCl}$ recommendations).

\section{CRP and Clinical Outcomes}

Of 780 patients with negative mRDT in the ePOCT arm, $50 \%$ (393) met criteria for CRP testing, that is, had tachypnea (respiratory rate $\geq 75$ th percentile for age and temperature; Supplementary Table 6) or chest indrawing (Figures 1 and 3; Table 4). No clinical failures occurred in the 10 patients with CRP values $\geq 80 \mathrm{mg} / \mathrm{L}$ or in the 16 patients with CRP values between 40 and $80 \mathrm{mg} / \mathrm{L}$ (Figure 3 and Table 4 ). The clinical course of the 13 patients with CRP $<40 \mathrm{mg} / \mathrm{L}$ on D0 who met clinical failure criteria were as follows: 2 were hospitalized secondarily with severe respiratory distress on D1 (Supplementary Table 5); 6 met clinical failure criteria at D3, of whom 5 recovered without antibiotic treatment by D7 (suggesting a viral respiratory infection); and the remaining 5 patients met clinical failure criteria at D7.

\section{Antibiotic and Salbutamol Treatment}

Overall, among mRDT-negative patients who received antibiotic treatment in the ePOCT arm, $0 \%(0 / 18)$ patients experienced clinical failure by D7 (Table 5) vs $7.1 \%$ (18/313) in the ALMANACH arm. Within the ALMANACH arm, patients who received antibiotic treatment at D0 had approximately a 2-fold risk of clinical failure compared to patients who did not receive antibiotics (RR, 1.81 [95\% CI, .98-3.32]). Within the ePOCT arm, there was no association between antibiotic treatment and clinical failure (Table 5). Salbutamol was prescribed as a home treatment for $17 \%(136 / 780)$ of patients in the ePOCT arm (based on a respiratory rate decrease after a salbutamol trial) and for 2\% (17/769) of patients in the ALMANACH arm (based on improvement of wheezing after a salbutamol trial). Within the ePOCT arm, there was no association between salbutamol treatment and clinical failure (Table 5).

\section{DISCUSSION}

We found that a 2-step algorithmic approach to inform antibiotic treatment (refined age-and temperature-based respiratory rate thresholds, followed by POC CRP), integrated into a tablet-based electronic decision algorithm (ePOCT), improved clinical outcomes among children with febrile illness and respiratory symptoms compared with the currently recommended WHO approach. Children managed using the CRP-informed strategy had a lower risk of clinical failure by D7, lower risk of developing severe symptoms by $\mathrm{D} 7$, and lower risk of severe adverse events (secondary hospitalizations or deaths) by D30. Using the CRP-informed strategy, antibiotic prescription at D0 was reduced substantially from $41 \%$ to $2 \%$.

To our knowledge, this is the first randomized clinical trial designed to evaluate a CRP-informed antibiotic prescription strategy in terms of clinical outcomes among children with febrile respiratory infections. To assess clinical outcomes was important as reported accuracies of CRP to detect bacterial pneumonia in children have been very heterogeneous $[9,16]$, largely due to the lack of an adequate reference standard [7]. Several clinical trials have been performed in adults showing similar clinical recovery to standard care [17], which led us to design our study as a noninferiority comparison. We chose to assess the impact of CRP testing within an overall disease management algorithm, rather than as a stand-alone intervention, as febrile children require evaluation of possible concomitant clinical problems. We were 


\begin{tabular}{|c|c|c|c|c|}
\hline Outcome & $\begin{array}{c}\text { ePOCT } \\
\% \text { (no./No.) }\end{array}$ & $\begin{array}{l}\text { ALMANACH } \\
\% \text { (no./No.) }\end{array}$ & $\begin{array}{l}\text { Risk Difference } \\
(95 \% \mathrm{Cl})\end{array}$ & $\begin{array}{l}\text { Risk Ratio } \\
(95 \% \mathrm{Cl})\end{array}$ \\
\hline \multicolumn{5}{|l|}{ Primary outcome } \\
\hline Clinical failure by day 7 & $2.9(25 / 865)$ & $4.8(41 / 854)$ & $-1.9(-3.7$ to -.1$)$ & $0.60(.37-.98)$ \\
\hline \multicolumn{5}{|l|}{ Secondary outcome } \\
\hline Antibiotic prescription at day 0 & $2.3(20 / 865)$ & $40.4(345 / 854)$ & $-38.0(-41.5$ to -34.6$)$ & $0.06(.04-.09)$ \\
\hline Antibiotic prescription by day $7^{a}$ & $6.8(59 / 865)$ & $43.8(374 / 854)$ & $-37.0(-40.7$ to -33.2$)$ & $0.16(.12-.20)$ \\
\hline Severe adverse events by day 30 & $0.5(4 / 865)$ & $1.5(13 / 854)$ & $-1.1(-2.0$ to -.1$)$ & $0.30(.10-.93)$ \\
\hline Secondary admissions & $0.5(4 / 865)$ & $1.4(12 / 854)$ & $-0.9(-1.8$ to 0$)$ & $0.33(.11-1.02)$ \\
\hline Deaths & $0(0 / 865)$ & $0.2(2 / 854)$ & $-0.2(-.5$ to .1$)$ & 0 (NA) \\
\hline
\end{tabular}

Results for the modified intention-to-treat analysis are available in Supplementary Table 1.

Abbreviations: ALMANACH, New Algorithm for Managing Childhood Illness Using Mobile Technology; Cl, confidence interval; NA, not applicable.

a Seventeen patients in the ePOCT arm and 20 patients in the ALMANACH arm received antibiotics by nonstudy physicians between day 0 and day 7 .

unable in this study to determine the relative contribution of each algorithm component (eg, salbutamol treatment, rehydration, targeted antibiotic prescription) to the overall improvement in clinical outcome and recommend that larger effectiveness studies be undertaken in the future. Several mechanisms could explain for the better clinical outcomes observed. First, an overreliance on antibiotic treatment may result in neglect of supportive measures for viral respiratory infections, such as salbutamol treatment for asthma. Salbutamol was indeed infrequently used in the ALMANACH arm (Table 5) [5]. We did not detect a significant association between salbutamol treatment and clinical failure in the ePOCT arm, but the study was likely underpowered to detect this association. Second, children may have experienced adverse effects of unnecessary antibiotic treatment. Our results are consistent with a more recent meta-analysis, which showed that the use of procalcitonin improved clinical outcomes in adults with acute respiratory infections [18].
In the present trial, the CRP-informed strategy was associated with an approximately 20 -fold reduction in antibiotic prescription. This is a substantial additional reduction compared with a recent trial in Vietnam, which reported a moderate reduction in antibiotic prescription (from $78 \%$ to $64 \%$ ) among patients with respiratory infections (including 287 children without tachypnea and chest indrawing) [19]. The difference may have resulted in part from an unguided use of POC CRP testing in the Vietnam study in combination with the application of a low cutoff ( $10 \mathrm{mg} / \mathrm{L}$ in children), and the use of CRP testing among patients with low-pretest probability of bacterial pneumonia [20]. A recent study in Norway found that, among febrile pediatric outpatients with respiratory infections, indiscriminate preconsultation CRP testing did not reduce antibiotic prescription rates when compared to physician-guided testing [21]. Two pediatric studies in high-income settings found no reduction in antibiotic prescription when using a very low procalcitonin

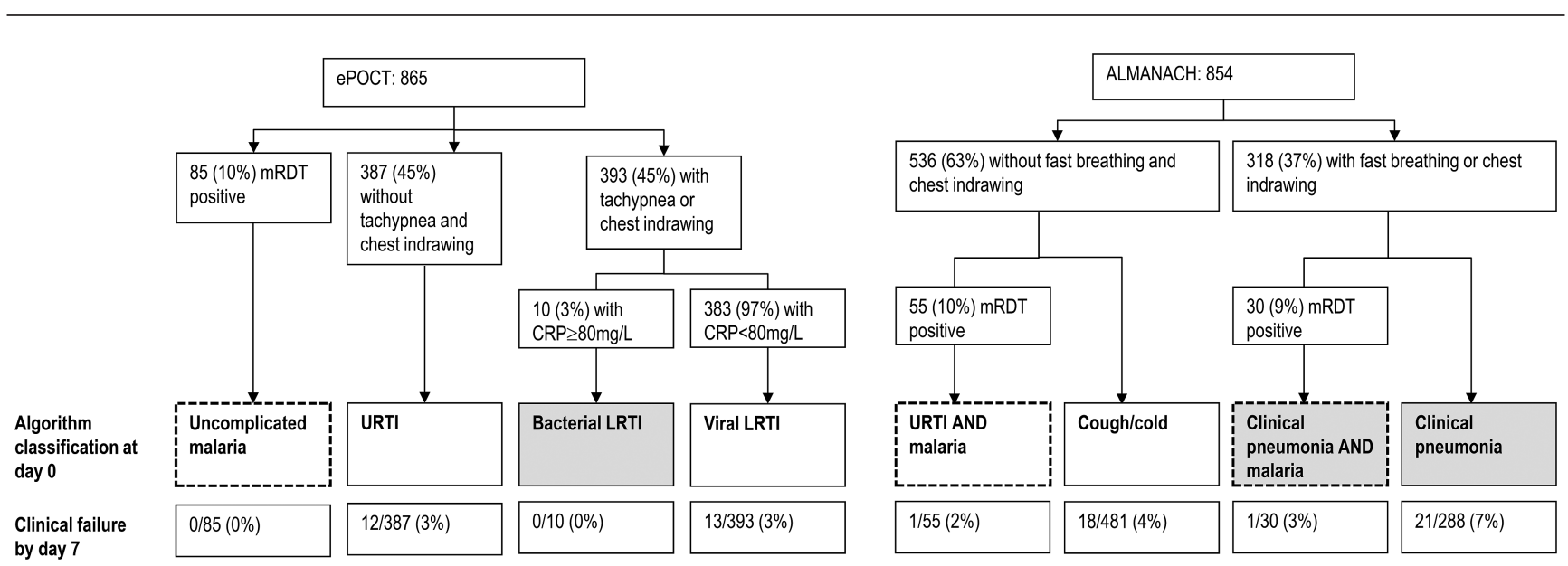

Figure 3. Decision algorithm including clinical classifications, point-of-care testing, and algorithm classifications. The diagnostic steps for each algorithm classification (see Figure 2), as well as the proportion of clinical failures within each algorithm classification, are displayed. Dotted outline: algorithm recommends antimalarial treatment; white: algorithm recommends symptomatic care; gray: algorithm recommends antibiotic treatment. The actual number of patients treated with antibiotics differed as patients had other concomitant classifications for which the algorithm recommended antibiotic treatment (see Table 4). Abbreviations: ALMANACH, New Algorithm for Managing Childhood IIIness Using Mobile Technology; CRP, C-reactive protein; LRTI, lower respiratory tract infection; mRDT, malaria rapid diagnostic test; URTI, upper respiratory tract infection. 


\begin{tabular}{|c|c|c|c|c|c|c|}
\hline \multirow[b]{2}{*}{$\begin{array}{l}\text { Algorithm Classification } \\
\text { at Day } 0\end{array}$} & \multicolumn{3}{|c|}{ ePOCT $(n=780)$} & \multicolumn{3}{|c|}{ ALMANACH $(n=769)$} \\
\hline & $\begin{array}{c}\text { Antibiotic } \\
\text { Prescription at Day 0 }\end{array}$ & $\begin{array}{c}\text { Antibiotic } \\
\text { Prescription by Day } 7\end{array}$ & $\begin{array}{c}\text { Clinical Failure by } \\
\text { Day } 7\end{array}$ & $\begin{array}{c}\text { Antibiotic Prescription } \\
\text { at Day } 0\end{array}$ & $\begin{array}{c}\text { Antibiotic Prescription } \\
\text { by Day } 7\end{array}$ & $\begin{array}{c}\text { Clinical Failure } \\
\text { by Day } 7\end{array}$ \\
\hline URTI & $0.5(2 / 387)^{a}$ & $5.4(21 / 387)$ & $3.1(12 / 387)$ & $4.6(22 / 481)^{a}$ & $10.4(50 / 481)$ & $3.7(18 / 481)$ \\
\hline LRTI & $1.5(6 / 393)^{\mathrm{a}}$ & $6.6(26 / 393)$ & $3.3(13 / 393)$ & NA & NA & NA \\
\hline \multicolumn{7}{|l|}{ CRP, mg/L } \\
\hline$<10$ & $1.2(3 / 256)^{\mathrm{a}}$ & $7.0(18 / 256)$ & $3.9(10 / 256)$ & $\ldots$ & $\ldots$ & $\ldots$ \\
\hline $10-39$ & $1.8(2 / 111)^{a}$ & $6.3(7 / 111)$ & $2.7(3 / 111)$ & $\ldots$ & $\ldots$ & $\ldots$ \\
\hline $40-79$ & $6.3(1 / 16)^{a}$ & $6.3(1 / 16)$ & $0(0 / 16)$ & $\ldots$ & $\ldots$ & $\ldots$ \\
\hline$\geq 80$ & $100(10 / 10)^{b}$ & $100(10 / 10)$ & $0(0 / 10)$ & NA & NA & NA \\
\hline Clinical pneumonia $^{c}$ & NA & NA & NA & $100(288 / 288)$ & $100(288 / 288)$ & $7.3(21 / 288)$ \\
\hline
\end{tabular}

Data are presented as \% (no./No.). No statistical comparison was made between the 2 study arms because the respiratory rate cutoffs used to define the URTI classification were different between the ePOCT and ALMANACH arms (see Figure 1 and Supplementary Table 6).

Abbreviations: ALMANACH, New Algorithm for Managing Childhood Illness Using Mobile Technology; CRP, C-reactive protein; LRTI, lower respiratory tract infection; NA, not applicable; URTI, upper respiratory tract infection.

${ }^{a}$ Antibiotic treatment was prescribed for concomitant bacterial infections (skin infections, dysentery, and ear infections).

${ }^{b}$ Six of the 10 patients with CRP values of $\geq 80 \mathrm{mg} / \mathrm{L}$ in the ePOCT arm would have been classified as pneumonia per the World Health Organization Integrated Management of Childhood IIIness (IMCI) guidelines, ie, they had tachypnea per IMCl criteria.

${ }^{\circ}$ Clinical pneumonia refers to the ALMANACH pneumonia classification based on respiratory rate (see Figure 1).

cutoff to recommend antibiotic prescription; physician compliance was poor $[22,23]$. This contrasts with European findings in adults [24], but is in line with a more recent US-based effectiveness study [25]. Together, these indicate that CRP testing is not beneficial when performed on the entire population of febrile patients but can be effective when integrated in a clinical management algorithm. Biomarker tests with poor specificity should only be used to inform diagnosis in patients with a reasonable (pretest) probability of bacterial pneumonia. To result in strong diagnostic performance, clinical predictors with high sensitivity should be employed as an initial "rule-out" step [8] before CRP is used as an additional second "rule-in" step with a high-enough cutoff value to maintain specificity [26]. Many of the patients in our study had symptoms in addition to cough that required specific medical attention. The integration of a "respiratory package" into an overall disease management tool supports an integrated approach to the management of children with fever.

Very few children in our trial met CRP criteria for antibiotic treatment. This raises the question of whether CRP testing (or antibiotic treatment) is actually useful at all in children with nonsevere respiratory infection. Indeed, a randomized controlled trial in Pakistan found placebo to be noninferior to amoxicillin in the treatment of children $<5$ years of age with WHO-defined pneumonia [4]. However, epidemiologic data suggest that, even with adequate vaccine coverage, a small proportion of children with respiratory infections continue to suffer from bacterial pneumonia [7]. The study in Pakistan may have been underpowered to detect differences in unfavorable outcomes and did not systematically assess severe adverse events. In the present study, approximately half of secondary hospitalizations occurred in children who had received an antibiotic on D0. The majority of these hospitalizations were likely due to viral infections. This illustrates that it is remains very difficult to predict poor outcome at an early stage of the episode. Supportive care (such as oxygen therapy and rehydration) is important for children with respiratory infections.

This study has some limitations. CRP testing was not used to inform management among children with severe disease or severe malnutrition requiring hospital referral. We hypothesized that children with severe disease, which are rare in primary care, likely benefit from antibiotic treatment [3], and that children with severe malnutrition have distinct immune responses

Table 5. Clinical Failure and Treatments Prescribed at Day 0 for Patients With Negative Malaria Rapid Diagnostic Test in the eP0CT ( $n=780$ )

Clinical Failure by Day 7

\begin{tabular}{lccc}
\cline { 2 - 4 } ePOCT Arm Treatment & Yes & No & RR (95\% Cl \\
\hline Antibiotic treatment at day 0 & $0 \%(0 / 18)$ & $3.3 \%(25 / 762)$ & $0(N A)$ \\
Salbutamol prescribed for home treatment & $2.2 \%(3 / 136)$ & $3.4 \%(22 / 644)$ & $0.65(.20-2.12)$ \\
\hline
\end{tabular}

Data are presented as \% (no./No.) unless otherwise indicated.

Abbreviations: $\mathrm{Cl}$, confidence interval; $\mathrm{NA}$, not applicable; RR, respiratory rate. 
that have not been adequately characterized [27]. Respiratory rate-based detection of children at risk for bacterial pneumonia is recommended by WHO for use in low-resource settings, and is inferior to complex clinical assessment as available in high-resource settings. We would not expect the same benefit in terms of clinical outcome in such settings; however, a benefit in terms of antibiotic prescription is more likely. The generalizability of this study is also limited by the fact that it was a single-country, urban study with low HIV prevalence, moderate malaria prevalence, and high vaccine coverage. Ultimately, the potential impact of this CRP-informed strategy will depend on clinician compliance with the decision algorithm. Further effectiveness studies should be performed to investigate factors associated with compliance and adoption. These studies should also reassess effects on secondary hospitalizations and deaths; this study was underpowered to detect a difference in these outcomes. Previous studies conducted among general practitioners suggest that POC CRP is most effective when accompanied by educational components [28]; the deployment of clinical algorithms provides unique opportunities in terms of continuous education.

In conclusion, this trial provides first evidence that POC CRP-informed strategies, integrated into an overall disease management algorithm, have the potential to improve clinical outcomes in children with acute respiratory infections in low-resource settings, while substantially improving the rational use of antibiotics. This trial was unique in that it included children at significant risk of bacterial pneumonia. Given the morbidity and mortality attributable to respiratory infections in developing countries, and the high-volume antibiotic overuse, our findings have significant implications for child survival and antimicrobial stewardship strategies. Future research efforts should focus on testing the effect in high-resource settings, and determining effectiveness in low-resource settings with larger trials.

\section{Supplementary Data}

Supplementary materials are available at Clinical Infectious Diseases online. Consisting of data provided by the authors to benefit the reader, the posted materials are not copyedited and are the sole responsibility of the authors, so questions or comments should be addressed to the corresponding author.

\section{Notes}

Author contributions. K. K., B. G., and V. D. jointly designed the study and applied for funding. Data were collected by J. M., J. S., H. T., and Z. S., with supervision from F. K. and K. K. W. S. and F. K. coordinated work with the study sites. T. M. was responsible for laboratory work. K. K. and V. D. led the statistical analysis and had full access to the database. The first and subsequent versions of the manuscript were written by K. K., edited by B. G. and V. D., and revised critically by all authors. All authors participated in data collection, data analysis, and data interpretation, and have approved the final manuscript. The corresponding author attests that all listed authors meet authorship criteria and that no others meeting the criteria have been omitted. The corresponding author had full access to all trial data and assumes final responsibility for the decision to submit for publication.

Acknowledgments. The authors thank the parents and children who agreed to participate in this study, the entire ePOCT team for their work in carrying out the trial, and the participating and collaborating hospitals, health centers, and dispensaries for their support. They also thank Clotilde Rambaud-Althaus and Alain Gervaix for their input on the development of the content of ePOCT; Thomas Routen for programming the ePOCT algorithm into the Mangologic software; and the data and safety monitoring board members for supervising the trial (Davidson Hamer, Rodrick Kisenge, and Mario Gehri).

Disclaimer. No funding body had any role in protocol design, data collection, analysis, interpretation, or writing of the manuscript. There was no monetary payment from any agency or pharmaceutical company for the writing of this manuscript.

Financial support. This work was supported by the Swiss National Science Foundation's R4D Program (grant number IZ01Z0_146896) and the Thrasher Research Fund (grant number 12588). The C-reactive protein tests used in this study were provided free of charge from bioMérieux.

Potential conflicts of interest. All authors: No potential conflicts. All authors have submitted the ICMJE Form for Disclosure of Potential Conflicts of Interest. Conflicts that the editors consider relevant to the content of the manuscript have been disclosed.

\section{References}

1. Risk R, Naismith H, Burnett A, Moore SE, Cham M, Unger S. Rational prescribing in paediatrics in a resource-limited setting. Arch Dis Child 2013; 98:503-9.

2. Liu L, Oza S, Hogan D, et al. Global, regional, and national causes of child mortality in 2000-13, with projections to inform post-2015 priorities: an updated systematic analysis. Lancet 2015; 385:430-40.

3. Shao AF, Rambaud-Althaus C, Samaka J, et al. New algorithm for managing childhood illness using mobile technology (ALMANACH): a controlled non-inferiority study on clinical outcome and antibiotic use in Tanzania. PLoS One 2015; 10:e0132316.

4. Hazir T, Nisar YB, Abbasi S, et al. Comparison of oral amoxicillin with placebo for the treatment of World Health Organization-defined nonsevere pneumonia in children aged 2-59 months: a multicenter, double-blind, randomized, placebo-controlled trial in Pakistan. Clin Infect Dis 2011; 52:293-300.

5. Nantanda R, Tumwine JK, Ndeezi G, Ostergaard MS. Asthma and pneumonia among children less than five years with acute respiratory symptoms in Mulago Hospital, Uganda: evidence of under-diagnosis of asthma. PLoS One 2013; 8:1-9.

6. World Health Organization. IMCI chart booklet. Report number 978924150682 3. Geneva, Switzerland: WHO, 2014.

7. McIntosh K. Community-acquired pneumonia in children. N Engl J Med 2002; 346:429-37.

8. Rambaud-Althaus C, Althaus F, Genton B, D’Acremont V. Clinical features for diagnosis of pneumonia in children younger than 5 years: a systematic review and meta-analysis. Lancet Infect Dis 2015; 15:439-50.

9. Flood RG, Badik J, Aronoff SC. The utility of serum C-reactive protein in differentiating bacterial from nonbacterial pneumonia in children. Pediatr Infect Dis J 2008; 27:95-9.

10. Brink AJ, Van Wyk J, Moodley VM, et al. The role of appropriate diagnostic testing in acute respiratory tract infections: an antibiotic stewardship strategy to minimise diagnostic uncertainty in primary care. S Afr Med J 2016; 106:30-7.

11. Keitel K, Kagoro F, Samaka J, et al. A novel electronic algorithm using host biomarker point-of-care tests for the management of febrile illnesses in Tanzanian children (e-POCT): a randomized, controlled non-inferiority trial. PLoS Med 2017; 14:e1002411.

12. D’Acremont V, Kilowoko M, Kyungu E, et al. Beyond malaria-causes of fever in outpatient Tanzanian children. N Engl J Med 2014; 370:809-17.

13. Nijman RG, Thompson M, van Veen M, Perera R, Moll HA, Oostenbrink R. Derivation and validation of age and temperature specific reference values and centile charts to predict lower respiratory tract infection in children with fever: prospective observational study. BMJ 2012; 345:e4224.

14. Thompson M, Harnden A, Perera R, et al. Deriving temperature and age appropriate heart rate centiles for children with acute infections. Arch Dis Child 2009; 94:361-5.

15. World Health Organization. WHO child growth standards. Report number 9789241546935. Geneva, Switzerland: WHO, 2006. 
16. Higdon MM, Le T, O'Brien KL, et al. Association of C-reactive protein with bacterial and respiratory syncytial virus-associated pneumonia among children aged $<5$ years in the PERCH study. Clin Infect Dis 2017; 64: S378-86.

17. Aabenhus R, Jensen J-US, Jorgensen KJ, Hrobjartsson A, Bjerrum L. Biomarkers as point-of-care tests to guide prescription of antibiotics in patients with acute respiratory infections in primary care. Cochrane Database Syst Rev 2014; 11:CD010130.

18. Schuetz P, Wirz Y, Sager R, et al. Effect of procalcitonin-guided antibiotic treatment on mortality in acute respiratory infections: a patient level meta-analysis. Lancet Infect Dis 2018; 18:95-107.

19. Do NTT, Ta NTD, Tran NTH, et al. Point-of-care C-reactive protein testing to reduce inappropriate use of antibiotics for non-severe acute respiratory infections in Vietnamese primary health care: a randomised controlled trial. Lancet Glob Health 2016; 4:e633-41.

20. Aabenhus R, Jensen JU. Biomarker-guided antibiotic use in primary care in resource-constrained environments. Lancet Glob Health 2016; 4:e586-7.

21. Rebnord IK, Sandvik H, Batman Mjelle A, Hunskaar S. Out-of-hours antibiotic prescription after screening with $\mathrm{C}$ reactive protein: a randomised controlled study. BMJ Open 2016; 6:1-7.
22. Baer G, Baumann P, Buettcher M, et al. Procalcitonin guidance to reduce antibiotic treatment of lower respiratory tract infection in children and adolescents (ProPAED): a randomized controlled trial. PLoS One 2013; 8:e68419.

23. Esposito S, Tagliabue C, Picciolli I, et al. Procalcitonin measurements for guiding antibiotic treatment in pediatric pneumonia. Respir Med 2011; 105:1939-45.

24. Albrich WC, Dusemund F, Bucher B, et al; ProREAL Study Team. Effectiveness and safety of procalcitonin-guided antibiotic therapy in lower respiratory tract infections in "real life": an international, multicenter poststudy survey (ProREAL). Arch Intern Med 2012; 172:715-22.

25. Huang DT, Yealy DM, Filbin MR, et al. Procalcitonin-guided use of antibiotics for lower respiratory tract infection. N Engl J Med 2018; 379:236-49.

26. Erdman LK, D’Acremont V, Hayford K, et al. Biomarkers of host response predict primary end-point radiological pneumonia in Tanzanian children with clinical pneumonia: a prospective cohort study. PLoS One 2015; 10:e137592.

27. Page AL, de Rekeneire N, Sayadi S, et al. Infections in children admitted with complicated severe acute malnutrition in Niger. PLoS One 2013; 8:e68699.

28. Little P, Stuart B, Francis N, et al; GRACE Consortium. Effects of internet-based training on antibiotic prescribing rates for acute respiratory-tract infections: a multinational, cluster, randomised, factorial, controlled trial. Lancet 2013 382:1175-82. 